\title{
SCREENING FOR BREAST CANCER
}

\author{
R W Blamey, A R M Wilson, J Patnick, J M Dixon
}

The aim of screening is to detect breast cancer when it is small and before it has had the chance to spread
Lack of knowledge of the pathogenesis of breast cancer means that primary prevention is currently a distant prospect. Screening represents an alternative approach to try to reduce mortality from breast cancer.

\section{Methods of screening}

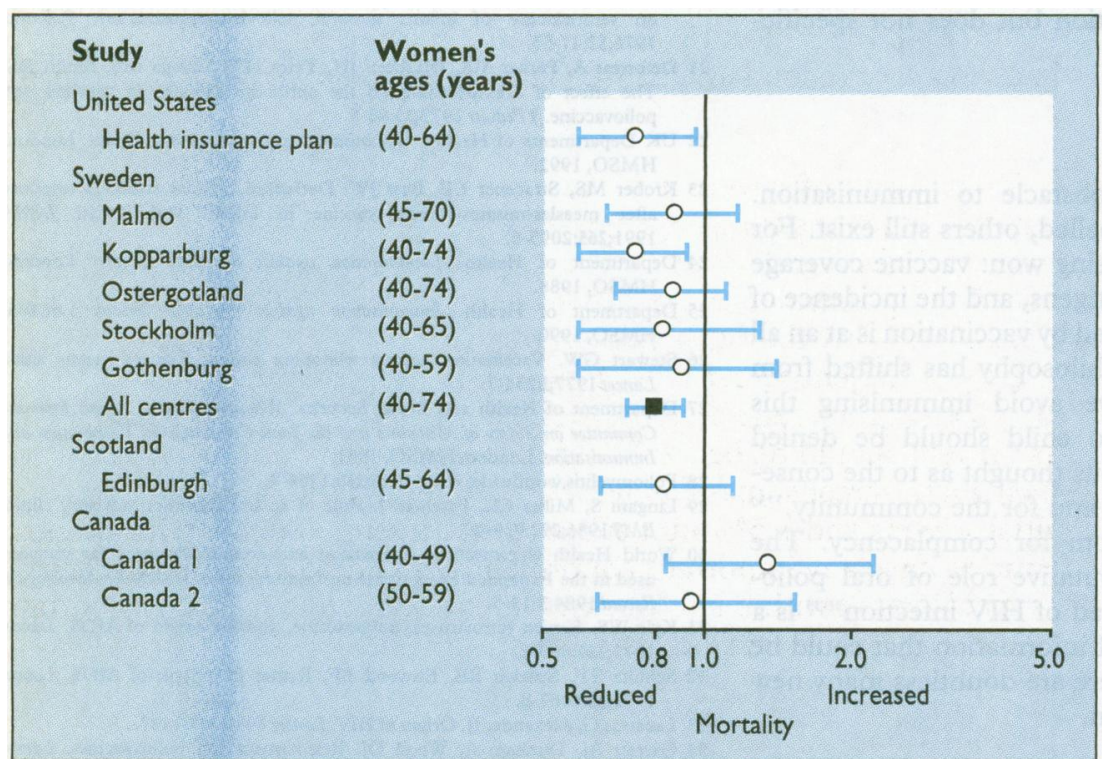

Summary of 7-12 years mortality data from randomised trials of breast cancer screening. Points and lines represent absolute change in mortality and confidence interval.
There is no evidence that clinical examination, breast ultrasonography, and teaching self examination of the breast are effective tools in screening for breast cancer. Randomised controlled trials have shown, however, that screening by mammography can significantly reduce mortality from breast cancer. Mortality can be reduced by up to $40 \%$ in women who attend for screening, and the benefit is greatest in women aged over 50 . Recently published data from the combined Swedish trials showed an overall reduction in breast cancer mortality of $29 \%$ during 12 years of follow up in women aged over 50 who were invited to attend screening and a $13 \%$ reduction in younger women.

Screening tests should be simple to apply, cheap, easy to perform, easy and unambiguous to interpret, and able to identify women with disease and exclude those without disease. Mammography is expensive; it requires high technology machinery, special film and dedicated processing, and highly trained radiologists to interpret the films; and it detects only $95 \%$ of all breast cancers. It is, however, the best screening tool available for detecting breast cancer and is the only screening method for any malignancy which has been shown to be of value in randomised trials.

\section{Organisational aspects of screening}

Over $70 \%$ of the target population must accept the invitation to participate if a screening programme is to significantly reduce mortality. If fewer participate the costs per life year saved rises and, although some will clearly benefit, the cost effectiveness of the programme comes into question. To achieve this, accurate lists of names, ages, and current addresses are required. Factors affecting attendance for screening include encouragement to attend by patients' general practitioners, knowledge about the screening programme, and the views of family and friends. Screening programmes should include both the initial screening process and assessment of any abnormality detected.

Standards should be set to ensure quality assurance at each stage of the screening process. Protocols should include referral for treatment to teams experienced in the management of breast disease. Specific training and programmes of continuing education related to screening should be mandatory for all professionals involved in the programme. Regular audit and review of both individual and programme results are essential. 


\section{Age range}

Current data indicate that the reduction in mortality is greatest in women aged 50-70. Although data from the combined Swedish trials show a reduction in mortality in younger women, the reduction is not significant and is delayed by at least eight years. Currently, therefore, there is no evidence to support screening of women aged under 50.

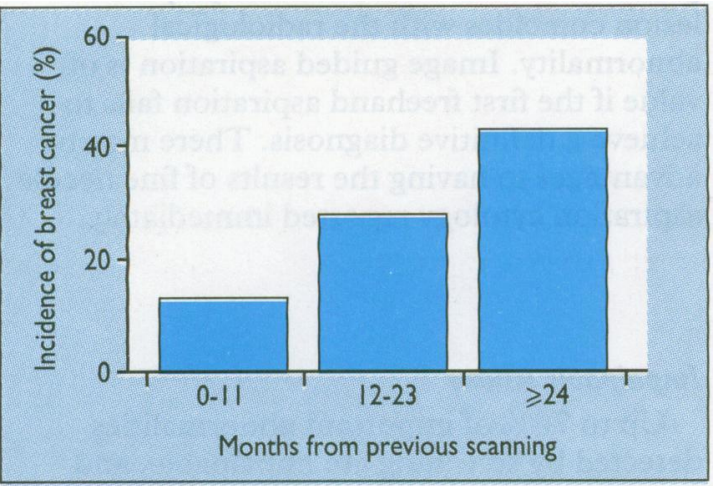

Rates of interval cancer after a negative screen in women aged 50-69.

\section{The basic screen}

\section{Detection of breast cancer in women aged 50-64 after an initial screen}

Initial screen

No of women

Recalled for assessment

Biopsy required

10000

$300-400$

Breast cancer detected

\section{Assessment}

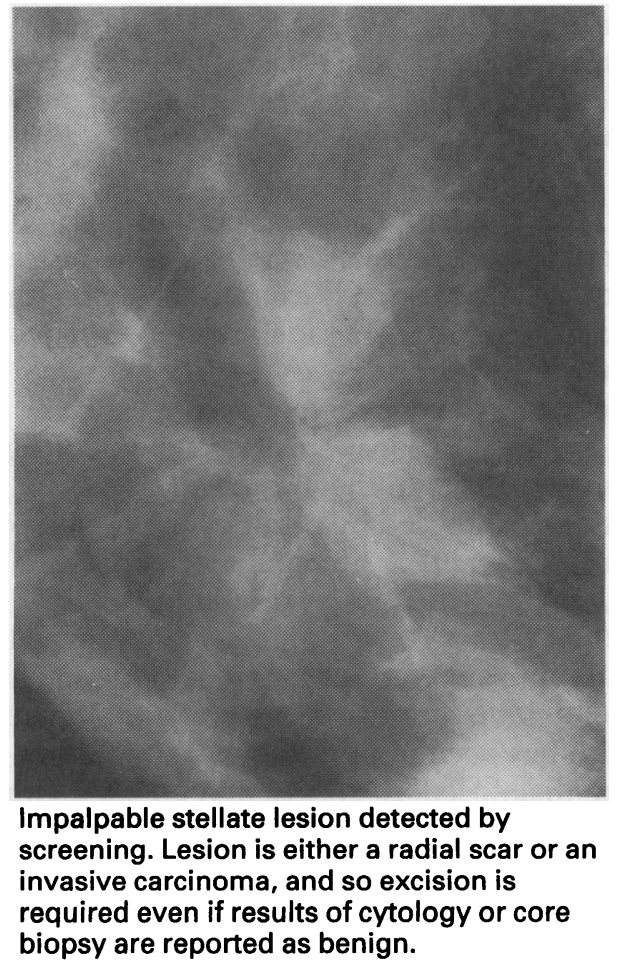

\section{Frequency of screening}

The most appropriate interval between mammographic screens has yet to be determined. From a cost-benefit point of view, screening once every three years for women aged 50-64 appears to be the optimal screening policy. However, the rate of interval cancer climbs rapidly between the second and third years after the initial screen, suggesting that a three year interval is too long. The best frequency for screening is probably between 18 months and two years.

\section{Number of mammographic views}

Whether patients should be screened initially by a single oblique mammogram or have two view mammography is not clear. Proponents of two view mammography argue that the extra expense of taking two $x$ ray pictures is offset by an increase in the rate of detecting breast cancer and a reduction in recall rates. Two views do, however, double the radiation dose, and there is insufficient evidence to show whether the extra view significantly reduces recall rates or rates of cancer detection. For second and subsequent screens a single view is sufficient.

The first part of screening is the basic screen. A radiologist is responsible for ensuring appropriate levels of sensitivity and specificity. Among women aged 50-64, at least 50 cancers should be detected for every 10000 attenders at an initial screen, reflecting the prevalence of cancer (currently the British programme detects over 60 per 10000 ). At the subsequent screens 35 cancers should be identified for every 10000 attenders, reflecting the incidence of cancer. Recall rates for assessment should be below $7 \%$ after initial screens and 3\% after subsequent screens; many units are recalling fewer than $3.5 \%$ after initial screens and $1.5 \%$ after subsequent screens. Women who are not found to have any important abnormality should be informed of their "normal" result within two weeks. Patients judged to have an important abnormality require further assessment.

There are two end points to assessment: no important abnormality or a diagnosis of breast cancer. Assessment requires further imaging and sometimes also clinical examination and cytology (triple assessment). Assessments are best performed by a dedicated assessment team consisting of an experienced radiologist, surgeon, and pathologist supported by a breast care nurse.

About two thirds of screen detected abnormalities are shown to be unimportant on further mammographic or ultrasound scanning. Counselling from a breast care nurse may be useful for women with a normal outcome, and it is mandatory for women who require further assessment or treatment for suspected or proved malignancy. In patients with important lesions the aim is to achieve a specific diagnosis by fine needle aspiration cytology or core needle biopsy. When the results of all investigations are available they are discussed by the multidisciplinary team, and a management decision is taken. Fine needle aspiration cytology can confirm a benign process and can definitively establish a malignancy, allowing a full discussion with a patient of the diagnosis and treatment options. Any surgery thereafter can be definitive. A biopsy should be performed if there is a suspicion of malignancy on either radiological or clinical grounds even when the results of cytology or core biopsy are benign. 


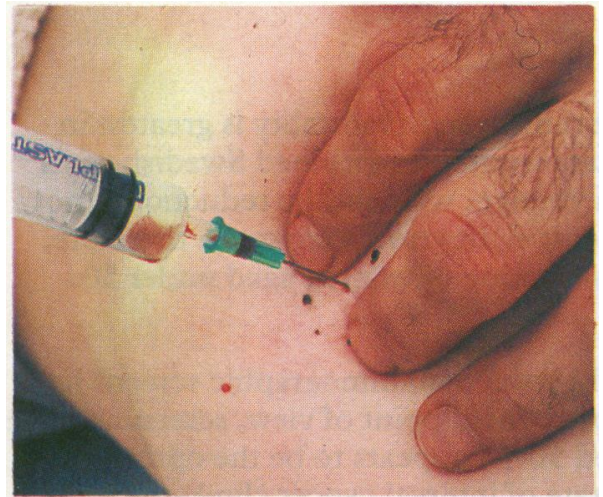

Fine needle aspiration: performed freehand (above) and guided by untrasound image (right).
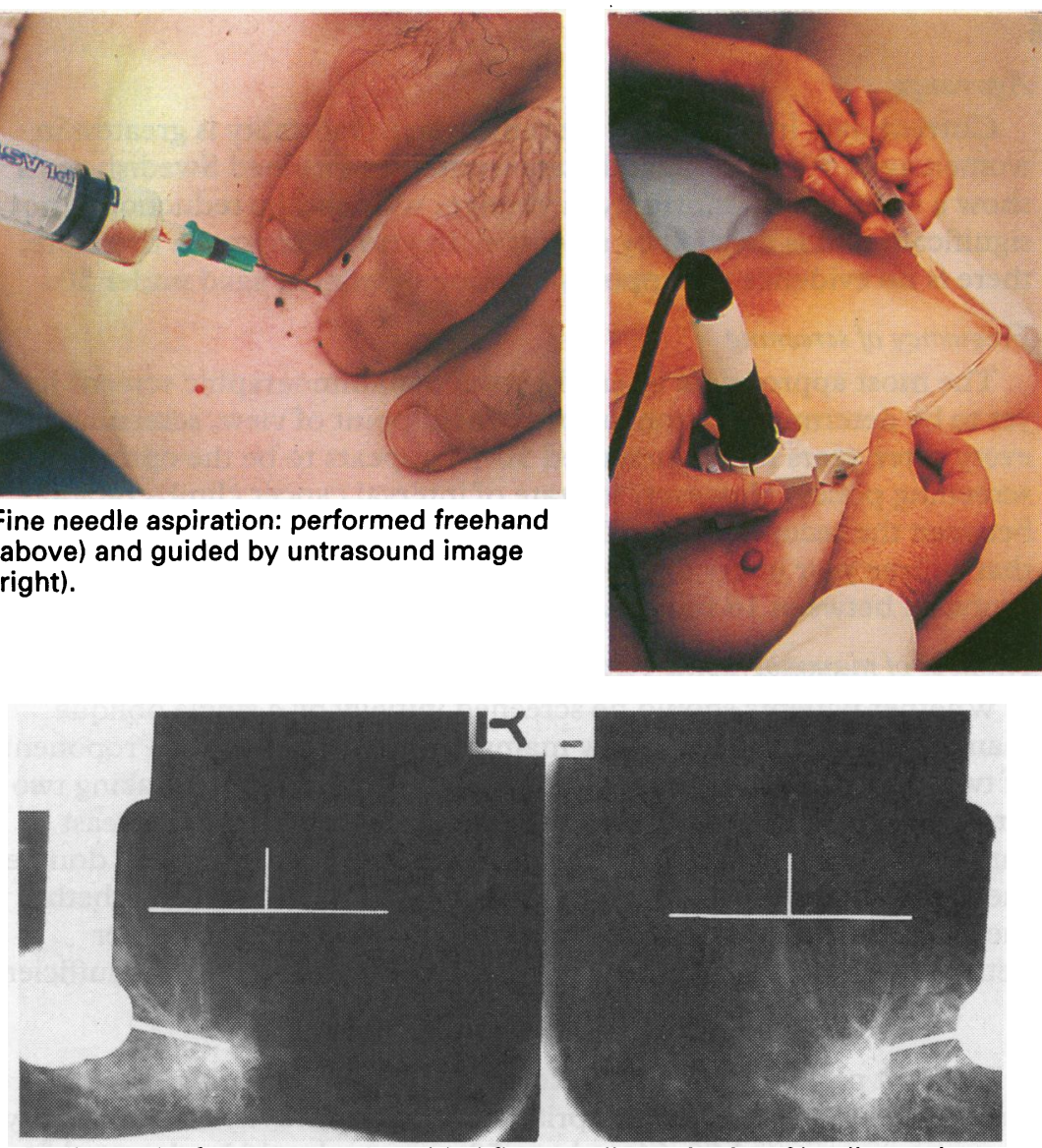

Radiographs for stereotactic guided fine needle aspiration. Needle can be seen penetrating the lesion.

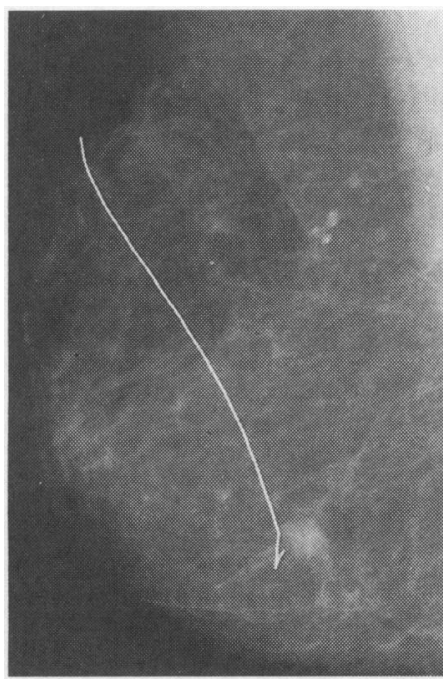

Mammogram after placement of hooked wire adjacent to mammographic lesion.

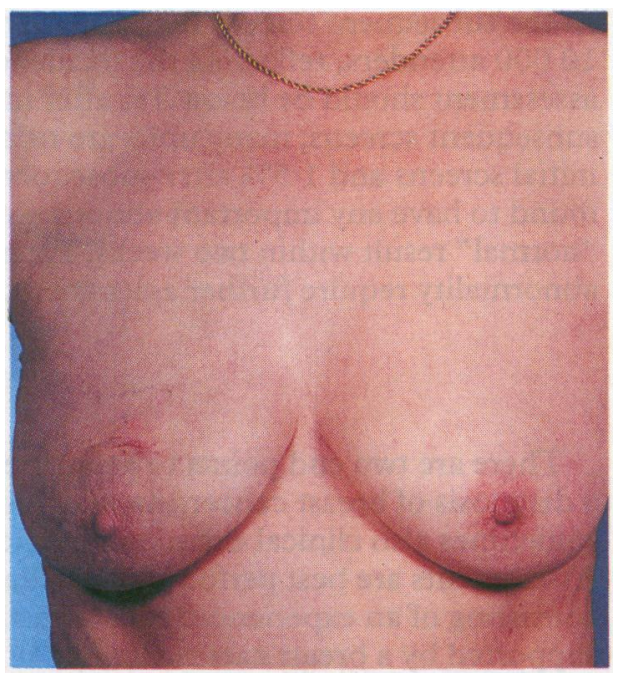

Cosmetic result of recent diagnostic excision biopsy-small scar and no loss of tissue.

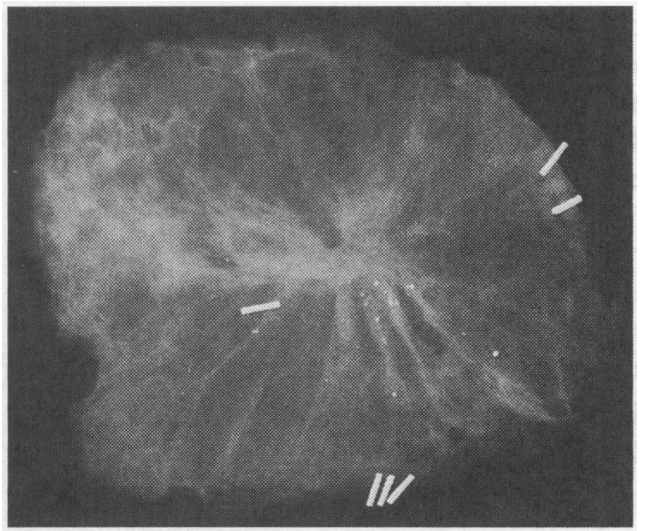

Specimen radiograph of therapeutic excision showing wide clearance margins around impalpable lesion. Ligaclips aid orientation-1 anterior, 2 medial, 3 inferior.

\section{Palpable lesions}

Fine needle aspiration of palpable lesions is usually carried out freehand or can be image guided if there is doubt that the palpable lesion coincides with the radiological abnormality. Image guided aspiration is of value if the first freehand aspiration fails to achieve a definitive diagnosis. There may be advantages to having the results of fine needle aspiration cytology reported immediately.

\section{Impalpable lesions}

Up to $70 \%$ of important abnormalities detected by screening are impalpable, and image guided fine needle aspiration is necessary. Impalpable lesions may be localised by ultrasonography if visible on this modality or by mammography. Ultrasonography is more accurate, quicker, easier to perform, cheaper, and associated with less patient discomfort. Some impalpable lesionsincluding most microcalcifications, several architectural disturbances, and some stellate lesions-are not visible on ultrasound scans and require $x$ ray guided needle aspiration. Stereotaxis is the most accurate of the $x$ ray guided techniques and should be performed with a 22 or 23 gauge needle of $9 \mathrm{~cm}$ length.

\section{Wire localisation biopsy and excision}

Ultrasonography and stereotaxis can facilitate surgery of impalpable lesions that require excision by allowing placement of a hooked wire adjacent to the mammographic lesion. The surgeon can follow the wire when making the incision and then excise breast tissue adjacent to the hook. Accurate placement of the wire is essential, and a variety of wire systems are available.

If the procedure is being performed to establish a diagnosis, a small representative portion of the lesion is excised through a small incision, so leaving a satisfactory cosmetic result if the lesion proves to be benign (according to surgical quality assurance guidelines the biopsy specimen should weigh less than $20 \mathrm{~g}$ ). In therapeutic excisions (lesions diagnosed by cytology or core biopsy to be malignant or highly suspicious of malignancy) the lesions should be widely excised with a 10-15 mm margin of normal tissue. Intraoperative specimen radiology is essential, both to check that the impalpable lesion has been removed and, if cancer has been diagnosed, to ensure that an adequate wide local excision has been performed. 


\section{Histological types of screen detected and symptomatic breast cancers

$\begin{array}{lcc}\text { Type } & \begin{array}{c}\text { Screen detected } \\ \text { carcinoma }\end{array} & \begin{array}{c}\text { Symptomatic } \\ \text { carcinoma }\end{array} \\ \text { Non-invasive } & 17 \% & 3 \% \\ \text { Invasive: } & 83 \% & 97 \% \\ \text { Special type } & 27 \% & 12 \% \\ \text { No special type } & 56 \% & 85 \%\end{array}$

*These have a better prognosis than cancers of no special type and include invasive tubular, cribriform, medullary, mucoid, papillary, and microinvasive cancers.

\section{Characteristics of screen detected cancers}

Compared with symptomatic cancers, screen detected cancers are smaller and more likely to be non-invasive (in situ), while any invasive cancers detected are more likely to be better differentiated, of special type, and node negative. The ability of screening to influence mortality from breast cancer indicates that early diagnosis identifies breast cancers at an earlier stage in their evolution when the chances of metastatic disease being present is smaller.

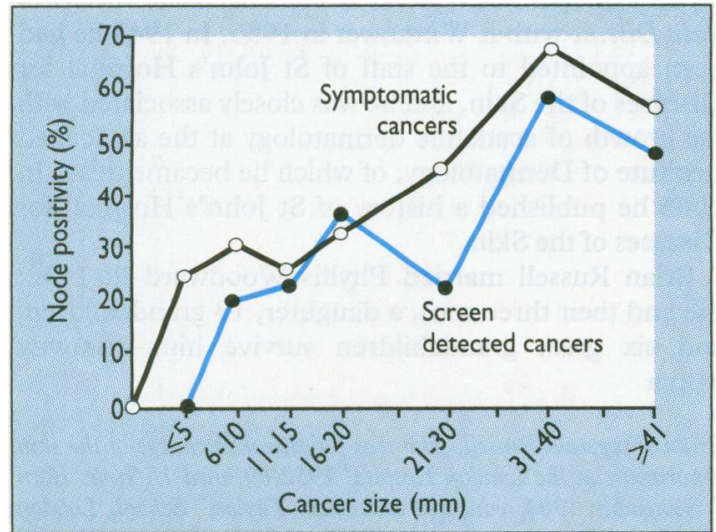

Relation between node positivity and tumour size for screen detected and symptomatic breast cancers.
Observed and expected detection of invasive cancer at initial screening of Swedish women aged 50-69

\begin{tabular}{cc} 
No of cancers detected \\
\hline $\begin{array}{cc}\text { Observed } & \text { Expected } \\
291 & 321.6\end{array}$
\end{tabular}

\section{Psychological morbidity induced by screening}

No increase in anxiety has been found in women invited to attend breast screening. There does appear to be a short term increase in anxiety associated with recall for assessment, but, by three months after attending for assessment, women who are shown to have no important abnormality (false positives) are no more anxious than control women. It has been suggested that the excess years as a breast cancer patient caused by a cancer being diagnosed earlier might diminish a patient's quality of life, but the psychological morbidity in women with screen detected breast cancer has been reported to be similar to or less than that in age matched controls.

\section{Risks of mammography}

It has been calculated that for every two million women aged over 50 who have been screened by means of a single mammogram one extra cancer a year after 10 years may be caused by the radiation delivered to the breast. Compared with an incidence of breast cancer that approaches 2000 in every million women aged 60 , this risk is very small.

\section{Overdiagnosis of breast cancer}

Some of the small, well differentiated, invasive cancers and some in situ cancers that are detected by screening would almost certainly not have caused symptoms during the patient's lifetime. However, results from the Swedish randomised trials indicate that the number of breast cancers that are being overdiagnosed by screening is small.

\section{Unnecessary biopsies}

A proportion of women who undergo biopsy will be found not to have cancer, but in Britain the number of women undergoing a biopsy for benign disease is small. The proportion of benign biopsies performed in a screening programme should be monitored and compared with that in an unscreened group of women of the same age. Women who require biopsy are likely to be extremely anxious, but there is no evidence that this anxiety is sustained if the results are benign.

J M Dixon acknowledges the support of the Cancer Research Campaign. Photographs were prepared by Mr D Dirom, Medical Illustration Unit, University of Edinburgh. The sources of the data presented in illustrations are: J M Dixon and J R C Sainsbury, Handbook of Diseases of the Breast (Churchill Livingstone) 1993:86 for the graph of results of trials of screening; L Tabar et al, Brf Cancer 1987;55:547-51 for the graph of rates of interval cancers between screens; T J Anderson et al, Br f Cancer 1991;64:108-13 for the graph of node positivity and cancer size for screen detected and symptomatic cancers; and N E Day, Br Med Bull 1991;47:400-17 (copyright British Council) for the table of observed and expected detection of cancer by screening. The data are reproduced with permission of the journals or copyright holders.

$\mathbf{R}$ Blamey is professor of surgical science and A R $\mathbf{M}$ Wilson is consultant radiologist at Nottingham City Hospital; Julietta Patnick is national coordinator of the NHS Breast Screening Programme, Sheffield; and J M Dixon is senior lecturer in surgery at Edinburgh Royal Infirmary and honorary consultant surgeon at Western General Hospital, Edinburgh.

The ABC of Breast Diseases has been edited by J M Dixon. 